\title{
MERCANTILISME ET UTOPIE DANS LA «PRÉFACE » DE L'ANATOMIE DE LA MÉLANCOLIE DE ROBERT BURTON
}

\author{
Claire Crignon de Oliveira
}

Presses Universitaires de France | Revue de métaphysique et de morale

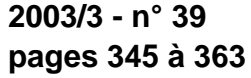

ISSN 0035-1571

Article disponible en ligne à l'adresse:

http://www.cairn.info/revue-de-metaphysique-et-de-morale-2003-3-page-345.htm

Pour citer cet article :

Crignon de Oliveira Claire, «Mercantilisme et utopie dans la «Préface » de L'Anatomie de la Mélancolie de Robert Burton »,

Revue de métaphysique et de morale, 2003/3 n³9, p. 345-363. DOI : 10.3917/rmm.033.0345

Distribution électronique Cairn.info pour Presses Universitaires de France.

(c) Presses Universitaires de France. Tous droits réservés pour tous pays.

La reproduction ou représentation de cet article, notamment par photocopie, n'est autorisée que dans les limites des conditions générales d'utilisation du site ou, le cas échéant, des conditions générales de la licence souscrite par votre établissement. Toute autre reproduction ou représentation, en tout ou partie, sous quelque forme et de quelque manière que ce soit, est interdite sauf accord préalable et écrit de l'éditeur, en dehors des cas prévus par la législation en vigueur en France. II est précisé que son stockage dans une base de données est également interdit. 


\section{Mercantilisme et utopie dans la «Préface » de L'Anatomie de la Mélancolie de Robert Burton}

RÉSUMÉ. - Si l'on s'accorde à voir dans l'ouvrage du clergyman mélancolique Robert Burton paru en 1621 une sorte d'aboutissement et de consécration de la mode mélancolique, l'on a toutefois tendance à négliger le fait que l'anatomiste utilise le discours médical et la tradition mélancolique pour attirer l'attention de ses contemporains sur l'existence d'un désordre qui se manifeste, au niveau de la collectivité, par une crise religieuse, politique, sociale et économique. C'est sous le patronage de l'un des premiers représentants du courant mercantiliste que Burton se place pour justifier l'emploi de la notion de "mélancolie politique » : celui de Giovanni Botero. La lecture de la «Préface » de L'Anatomie de la Mélancolie permet de constater l'existence d'une forte convergence entre les méthodes et les analyses des premiers mercantilistes anglais et le diagnostic de Robert Burton sur la crise économique que traverse l'Angleterre de Jacques $I^{e r}$.

Comment expliquer alors la coexistence dans le même texte de ce discours économique qui propose des réformes concrètes pour remédier à une situation de crise et d'un discours utopique qui préconise une forme autoritaire et disciplinaire de pouvoir comme remède à la mélancolie politique tout en affirmant le caractère chimérique et irréalisable de tout changement radical?

ABSTRACT. - The Anatomy of Melancholy (1621), written by the melancholic clergyman Robert Burton, is considered as a climax of the melancholic fashion. Nevertheless it is rarely underlined that Burton has recourse to the medical discourse and the melancholic tradition to draw his contemporaries's attention to a collective disorder which reveals itself in a religious, political, social and economical crisis. Robert Burton uses the authority of one of the first names associated to the mercantilist movement-Giovanni Botero - to justify his use of the notion of a "political melancholy". Reading the "Preface » of The Anatomy of Melancholy, one notices a strong convergence between the first English mercantilists's methods and analyses and Burton's diagnosis on the economic crisis which marks the reign of James the first. Thus is raised the question of a possible coexistence in the same text of the economic discourse, which suggests concrete reforms to face a crisis, with a utopian discourse which recommends an authoritative and disciplinary power as a remedy against political melancholy while asserting at the same time that any radical change is fanciful and impracticable. 
Homme de lettres et bibliothécaire du collège de Christchurch à Oxford jusqu'à sa mort en 1640, Robert Burton (1577-1640) a consacré toute sa vie à la rédaction d'un unique ouvrage sans cesse remis sur le métier ${ }^{1}$ dans lequel il se donne pour tâche d'anatomiser cette humeur mélancolique qui fut à la fois sa maîtresse, son égérie et son malin génie :

Mon objectif et ma tâche, dans le discours qui va suivre, est d'anatomiser cette humeur mélancolique en en suivant toutes les parties et caractéristiques [...] et de le faire philosophiquement, médicalement, pour en montrer les diverses causes, les divers symptômes et les divers moyens de la guérir, de sorte qu'il soit plus facile de l'éviter ${ }^{2}$.

S'opposant au fort courant de valorisation du tempérament mélancolique qui prévaut jusqu'à la fin de la Renaissance, courant qui tend à associer ce tempérament au génie des grands hommes, Burton met en garde ses contemporains contre le danger de cette mode mélancolique. La mélancolie est en effet d'abord pour l'anatomiste le nom d'une maladie, qui résulte du déséquilibre entre les quatre humeurs, leur harmonie étant pensée depuis la tradition hippocraticogalénique, comme la condition de la bonne santé de l'individu. Persuadé du caractère nocif de cette affection et du danger qu'elle représente pour l'ensemble de l'humanité, Burton choisit d'écrire sur la mélancolie pour se guérir lui-même de ce fléau, et aussi pour donner à ses concitoyens des moyens de lutter contre un mal par nature contagieux qui risque de gagner l'ensemble du corps politique si l'on ne fait rien pour en arrêter la progression ${ }^{3}$.

\section{D'UNE MÉLANCOLIE INDIVIDUELLE À UNE MÉLANCOLIE COLLECTIVE}

L'une des spécificités de l'ouvrage tient au fait que Burton, contrairement à ses contemporains, ne considère pas seulement la mélancolie comme une perturbation dont la dimension serait purement individuelle, mais qu'il lui donne une signification sociale et politique forte. Il établit en effet un lien entre la

1. La $1^{\text {re }}$ édition paraît en 1621 . Elle est suivie par quatre éditions du vivant de l'auteur au fil desquelles la taille du texte augmente considérablement. La dernière paraît en 1651 de manière posthume.

2. R. Burton, Anatomie de la Mélancolie, trad. B. Hoepffner, Paris, Corti, 2000, « Au Lecteur », p. 194. Voir aussi le titre complet de l'ouvrage : L'Anatomie de la Mélancolie. Ce qu'elle est, avec toutes les variétés, symptômes, pronostics et ses divers traitements. En trois parties, avec leurs sections, membres et sous sections respectifs, philosophiquement, médicalement, historiquement disséquée, par Démocrite Junior. Avec une Préface satirique conduisant au traité qui suit.

3. «Au Lecteur », p. 194-195 : «Étant donné, donc, qu'il s'agit d'une maladie très grave et fort répandue, je vois que prescrire les moyens de prévenir et de guérir une maladie aussi universelle, une maladie épidémique [...] est pour moi une façon de rendre service à tous et d'employer efficacement mon temps. » 
crise mélancolique qu'il a tenté de surmonter en rédigeant L'Anatomie de la Mélancolie, et la période d'instabilité économique qui caractérise le règne de Jacques $\mathrm{I}^{\text {er }}$, instabilité qui commence à prendre une ampleur inquiétante au moment même où paraît la première édition de L'Anatomie de la Mélancolie (1621). L'un des intérêts de la réflexion développée dans la «Préface » vient précisément de la relation qui s'établit entre la santé de l'individu et l'état de prospérité du corps politique auquel il appartient.

Pour mettre en évidence ce rapport, Burton convoque deux références : il revendique tout d'abord l'héritage des Lettres du Pseudo Hippocrate. Burton écrit la «Préface » en se servant d'un pseudonyme : celui de Democritus Junior. L'écriture de L'Anatomie de la Mélancolie répond à la nécessité de poursuivre la rédaction du traité sur l'origine de la bile noire que le philosophe Démocrite avait entrepris de rédiger. Les Lettres du Pseudo Hippocrate ${ }^{4}$ relatent comment les concitoyens de Démocrite, habitants de la ville d'Abdère, font appel au médecin Hippocrate, persuadés que Démocrite est devenu fou et affolés à l'idée que la folie de Démocrite puisse gagner l'ensemble des habitants de la cité d'Abdère. «Un très grave danger, Hippocrate, menace actuellement notre cité en menaçant un des nôtres », écrivent les Abdéritains à Hippocrate ${ }^{5}$. La référence à ce texte permet à Burton de rappeler qu'il n'est pas possible de guérir un individu (de sa maladie, de sa folie) tant que la cité à laquelle il appartient est en mauvaise santé.

Or justement, l'Angleterre de Jacques $\mathrm{I}^{\text {er }}$ est malade. Le règne de ce dernier est marqué par une très forte instabilité économique qui menace de déboucher sur une crise politique : la crise atteint son acmé entre 1620 et 1624 . La situation est si grave qu'une commission est nommée par le Parlement pour déterminer les causes du déclin du commerce et de la pénurie de monnaie qui frappe le pays ${ }^{6}$. La première édition de L'Anatomie de la Mélancolie paraît en 1621, au moment même où la situation se détériore. Conscient de la gravité de la crise, Burton exprime dans la «Préface» la nécessité d'une réforme ${ }^{7}$ : « La plus grande partie de notre commerce devrait être réformée ${ }^{8}$. »

4. Voir Sur le rire et la folie, préface, traduction et notes de Y. Hersant, Rivages poche, « Petite bibliothèque », Paris, 1995.

5. Ibid., p. 37.

6. Voir W. R. Mueller, The Anatomy of Robert Burton's England, University of California Press, Berkeley-Los Angeles, 1952, chap. IV, « The Economy », p. 35. Voir aussi F. Hermia DurHAM, « The Relations of the Crown to Trade under James I », Transactions of the Royal Historical Society, p. 236. La crise monétaire atteint son sommet en Angleterre dans les années 1622-1623.

7. Réforme qui, comme Burton prend bien soin de le préciser, ne met pas en cause la forme monarchique du gouvernement : « nous avons un roi sage, savant et pieux [...] des sénateurs de grande valeur, un clergé érudit, un peuple soumis, etc. Pourtant, dans cette abondance de roses, poussent quelques chardons, quelques mauvaises herbes et autres nuisances, qui troublent beaucoup la paix de ce corps politique, en éclipsent l'honneur et la gloire ; il faudrait les déraciner et nous réformer sans attendre » («Au Lecteur », p. 137).

8. «Au Lecteur», p. 145. 
Il choisit alors de décrire cette crise que traverse son pays par le biais du discours mélancolique en faisant de sa propre mélancolie un prétexte pour justifier un regard critique sur la société dans laquelle il vit, jugement critique qu'il applique aussi bien à la situation politique et religieuse de l'Angleterre, qu'à sa vie sociale, économique ou intellectuelle. Déterminé à montrer que « les royaumes, les provinces, les familles [sont] mélancoliques tout autant que les individus » (p. 112), Burton mentionne dans la « Préface » une seconde autorité pour confirmer son diagnostic : celle de l'Italien Giovanni Botero, l'un des fondateurs du mercantilisme, et renvoie en note au « De politia illustrium », liv. I.4 :

Les royaumes, les provinces et les corps politiques sont de même exposés et sujets à cette maladie, ce qui a été définitivement prouvé par Botero dans son étude sur la politique : comme dans les corps humains, dit-il, on y trouve [dans les corps politiques] diverses altérations provenant des humeurs, de sorte que de nombreuses et diverses maladies, conséquences d'une grande diversité de troubles, attaquent la communauté, et il est aisé de s'en rendre compte à l'aide de leurs symptômes spécifiques. [ « $\mathrm{Au}$ Lecteur », p. 123.]

Le passage auquel il fait ici référence n'est pourtant pas le livre I chap. $4 \mathrm{du}$ Discours sur la grandeur et la magnificence des villes de l'Italien Giovanni Botero, comme il est indiqué dans la note, mais le livre I, chap. 4 de De la raison d'État. Burton avait à sa disposition la traduction latine de 1602 : De Illustrium statu et politia ${ }^{9}$, qui réunit les deux textes de Botero (De la raison d'État et Discours sur la grandeur...), ce qui peut expliquer cette référence erronée. Mais les choses se compliquent encore car si la phrase à laquelle Burton fait référence se trouve bien dans la traduction latine du texte de Botero que Burton a consulté, elle n'est pas présente dans le texte original italien ${ }^{10}$.

Burton reprend aussi dans la «Préface » la distinction faite par Botero en tout début du livre I, chap. 4 (Des causes de la ruine des États) entre deux

9. Bodleian Douce 570, Ioannis Boteri Viri Clarissimi Tractacus Duo, Prior : De Illustrium statu \& politia, libris X. Posterior: De Origine urbium, earum excellentia \& augendi ratione, Libris III. Comprehensi, Ex Italico primum in Germanicum, atque exinde in Latinum trans lati, \& multorum memorabilium accessione, ac indice rerum \& verborum locupletissimo aucti, Auctore, M. Georgio Dravdio, cive Francofurtensi, Ursellis, 1602.

10. «Ac quemadmodum humanis corporibus variae accidunt mutationes, tum ab elementis, tum â corporis animique habitu, tum ab humorum temperatione, tum ab educatione alimentorumque varietate; ita quoque Rerumpublicarum conversiones sive in hanc sive in illam partem diversimode contingunt»: "Et de même que divers changements se produisent dans le corps humain, sous l'effet tantôt des éléments, tantôt des états du corps et de l'esprit, tantôt de l'équilibre des humeurs, tantôt de l'éducation et des changement du régime alimentaire; de même les transformations des Républiques surviennent de différentes manières, dans un sens ou dans l'autre. » (Je remercie Romain Descendre pour les informations données concernant Botero qui m'ont permis de démêler l'écheveau de la référence à Giovanni Botero dans la « Préface » de L'Anatomie.) 
sortes de causes susceptibles de produire la mort des œuvres de la nature : des causes internes et des causes externes. Il est donc fidèle au texte de Botero lorsqu'il dit, premièrement, que les corps politiques comme les corps naturels sont sujets à la corruption et à la maladie et, deuxièmement, qu'une double causalité permet de rendre compte de ces maladies du corps politique.

Néanmoins, Botero ne parle pas de mélancolie politique mais de maladie du corps politique, et d'autre part les causes internes et externes ne sont pas les mêmes chez les deux auteurs. Par exemple, alors que Botero comprend principalement dans les causes externes « les ruses, stratagèmes, et la puissance des ennemis », Burton lui ajoute d'autres facteurs, et en particulier la situation géographique, climatique, le « déclin commercial », les catastrophes naturelles (inondations, tempêtes) ou les maladies épidémiques, comme la peste. D’autre part, alors que les causes internes viennent essentiellement pour Botero de l'incapacité du prince, de sa cruauté, «[des] envies, dissensions, discordes et ambitions des grands, [de] la légèreté, l'instabilité, [de] la fureur de la populace, et [de] l'inclination des nobles et du peuple à un autre seigneur ${ }^{11}{ }^{\prime}$, Burton fait de la transformation ou modification du culte et de l'absence de crainte de Dieu la cause interne principale de la mélancolie politique.

Burton est fidèle à Botero dans la mesure où, pour lui, la mélancolie est la quintessence de toutes les maladies. Par conséquent, dire que le corps politique est susceptible d'être mélancolique, c'est simplement dire qu'il est sujet, comme tous les corps de l'univers, à la corruption. Mais la comparaison entre les deux textes permet de voir comment Burton réinterprète l'analogie classique du corps naturel et du corps politique à l'aune de la tradition mélancolique : ainsi la puissance des ennemis est pour lui un symptôme de la mélancolie politique (et pas seulement de la maladie en général) parce qu'elle a pour effet de provoquer la crainte chez les habitants qui sont menacés par l'invasion (les « habitants vivent dans une peur perpétuelle... »). De la même façon, les catastrophes naturelles suscitent un sentiment de terreur panique, et les maladies épidémiques ont encore souvent le statut de punition envoyée aux hommes pour leurs péchés, parce que la mélancolie représente aux yeux de Burton la condition de l'homme après la chute. Enfin et surtout, Burton intègre parmi les causes externes le déclin commercial : ce qui signifie que pour lui l'état de prospérité ou de déclin économique peut être considéré comme un symptôme de l'absence ou de la présence d'une forme politique de mélancolie.

Dire que le déclin commercial est un symptôme de mélancolie politique et pas seulement le symptôme d'une corruption du corps politique, facilite la tâche

11. Raison et Gouvernement d'Estat, en 10 livres, trad. Chappuys, Paris, G. Chaudiere, 1599, I, 4 : «Les causes de la ruine des États », p. 7-8. 
de Burton : d'une part, la référence à Botero lui permet de réaliser son projet qui consiste à montrer que tous les corps appartenant à l'univers sont susceptibles de souffrir de la mélancolie ${ }^{12}$ sans avoir à justifier plus avant l'existence d'une forme politique de mélancolie. Mais d'autre part, parler de «mélancolie » politique et pas simplement de «maladie» du corps politique permet d'établir une série de parallèles entre les symptômes de la crise que l'anatomiste traverse en tant qu'individu, et les symptômes de la crise politique que traverse selon lui l'Angleterre. Burton peut alors opposer l'image d'un État non mélancolique (en bonne santé) à l'image d'un État mélancolique (tableau qui correspond à la situation actuelle de l'Angleterre).

\section{LES SYMPTÔMES ÉCONOMIQUES DE LA CRISE : CONVERGENCE ENTRE LE PROPOS DE BURTON ET L'ANALYSE DES MERCANTILISTES}

Quels sont précisément les signes qui permettent de diagnostiquer l'absence de mélancolie au sein du corps politique selon Burton?

Car là où nous voyons que les gens sont polis, qu'ils obéissent à Dieu et aux princes, qu'ils sont judicieux, paisibles et calmes, riches et heureux, qu'ils prospèrent et vivent unis, en paix et en harmonie, une campagne bien labourée, de nombreuses villes bien construites et peuplées, où [...] les gens [...] vivent dans le bien et dans le bonheur, ce qui pour les hommes est l'objectif principal d'une communauté, ce qu'Aristote appelle le bien commun, $[\ldots]$ le pays n'est pas mélancolique ${ }^{13}[\ldots]$.

Pour Burton, l'idéal aristotélicien du bien commun s'identifie à la prospérité et à la bonne santé économique de l'État: la prospérité économique est selon lui la fin propre de la communauté politique, au lieu de rester comme chez Aristote, un simple moyen du bien vivre. Précisément, ce processus qui tend à intégrer en un système harmonieux les fins poursuivies par les marchands avec la fin propre de l'État est l'un des traits caractéristiques de la pensée des premiers mercantilistes anglais ${ }^{14}$. C'est une idée qui est exposée dès 1581 dans le texte intitulé Compendieux ou bref examen de quelques plaintes coutumières à divers de nos compatriotes ${ }^{15}$ (publié en 1581 par Thomas Marshe). Sous la forme d'un

12. «Au Lecteur », p. 122: "Cette mélancolie ne s'attache pas seulement aux humains, mais aussi aux plantes et aux êtres sensibles.»

13. L’Anatomie de la Mélancolie, «Au Lecteur», p. 123.

14. Voir H. DenIs, Histoire de la pensée économique. Paris, PUF, 1999 (1966).

15. A Discourse of the Commonweal of this Realm of England, Edited by Mary Dewar, The Folger Shakespeare Library, University Press of Virginia, 1969. 
dialogue, l'auteur montre comment l'intérêt collectif peut naître de l'affrontement des revendications opposées de différentes professions (le docteur, le gentilhomme, le fermier, et le bonnetier). La puissance de la Royauté est indissociable de la prospérité économique de ses habitants : sa majesté «ne peut manquer d'argent aussi longtemps que ses sujets en possèdent, mais serait impuissante et désarmée s'ils n'en avaient point, ils ne pourraient d'ailleurs en avoir s'il ne circulait plus d'espèces dans le royaume ${ }^{16}{ }$.

Burton ne vient pas d'une famille aisée ; il a travaillé comme vendeur pendant trois ans au marché d'Oxford ${ }^{17}$ et a donc une certaine expérience des pratiques commerciales. Il déplore en particulier l'absence de système de poids et de mesure unifié en Angleterre ${ }^{18}$. D'autre part, les fonctions de bibliothécaire qu'il a occupées au sein du collège de Christchurch lui ont donné une connaissance précise de l'abondante littérature pamphlétaire qui paraît dans les années 1620 sur ces questions économiques ${ }^{19}$ (Edward Misselden, Free Trade or the Meanes to Make Trade Florish ... ${ }^{20}$, Gérard de Malynes, A Treatise of the Canker of Englands Commonwealth, 1601 ; ou The Center of the Circle of Commerce or, A Refutation of a Treatise, Intituled The Circle of Commerce, or the Balance of Trade, 1623). Burton est convaincu que le développement du commerce et la prospérité économique constituent des remèdes possibles à la crise traversée par son pays.

Parallèlement, il montre dans la «Préface » que les symptômes économiques de la crise constituent autant de causes possibles de la mélancolie collective ou politique qui affecte le royaume des Stuarts. Voici quels sont pour lui les symptômes les plus frappants de la crise :

[...] si vous avez devant vous le spectacle de grands mécontentements, de griefs partagés par tous, de plaintes, de pauvreté, du barbarisme, de la mendicité, d'épidémies, de guerres, de rébellions, de séditions, de mutineries, de querelles, de l'oisiveté, d'émeutes, de l'épicurisme, de campagnes non labourées, ravagées, pleines de marécages, de marais, de déserts \& de cités déchues, de villes ruinées et pauvres, de villages dépeuplés, d'un peuple crasseux, laid et malpoli, ce royaume, ce pays est nécessairement chagrin et mélancolique, son corps est malade et il faut le guérir ${ }^{21}$.

16. Cité dans Pierre Deyon, Le Mercantilisme. Questions d'histoire, Flammarion, 1969, chap. I, p. 53.

17. Cf. J. Max PAtrick, « Robert Burton's Utopianism », Philosophical Quaterly, vol. 27, oct. 48, State University of Iowa, p. 346.

18. C'est là une des mesures qu'il préconise dans son utopie : « [...] les mesures seront les mêmes partout, homogénéisées à l'aide du primum mobile et du mouvement du soleil [...] » (p. 171-172).

19. Voir " Two lists of Burton's Books ", éd. S. Gibson et F. R. D. Needham, Oxford Bibliographical Society Proceedings and Papers, I, 1927, p. 222-246.

20. ... wherein the Causes of the Decay of Trade in the Kingdome are discovered, And the remedies also to remoove the same, are represented, the second edition with some addition, London printed by John Legatt, 1622.

21. «Au Lecteur », p. 124. (souligné par nous). 
Son diagnostic reprend les principaux travers dénoncés par ses prédécesseurs (L'Utopie de More, livre I) ou par ses contemporains (Bacon, Essais) : la distribution inégale des richesses et de la monnaie, et l'écart important entre le niveau de vie des plus riches et des plus pauvres. Burton met sur ce point en cause le mode de vie oisif de la noblesse et du clergé qui «mènent une vie délicate et splendide au sein de l'oisiveté », tandis que d'autres gagnent à peine de quoi subsister. Or non seulement l'oisiveté est selon Burton la cause première de la mélancolie, mais la pauvreté est aussi de son côté un facteur de mécontentement et une cause possible de rébellion ${ }^{22}$. Un État ne peut espérer être en bonne santé, quand toute une partie de la population est occupée à ne rien faire, et s'expose à ressentir les affres de la mélancolie, et que l'autre partie vit dans une misère telle, qu'elle risque de se soulever contre cet état de fait et de semer le désordre au sein du corps politique.

La seconde faiblesse énoncée dans le passage cité est liée au phénomène de dépopulation, conséquence selon Burton et ses contemporains du mouvement des enclosures qui s'est mis en place en Angleterre dès le $\mathrm{XV}^{\mathrm{e}}$ siècle ${ }^{23}$ : Thomas More ${ }^{24}$ explique dans le premier livre de L'Utopie comment la décision des propriétaires fonciers de clore les prés et les terres cultivables pour les transformer en pâturages a progressivement privé les paysans de la possibilité

22. L'Anatomie de la Mélancolie, p. 129. Burton renvoie à SALluste, Conjuration de Catilina, $\S 37$ : «Ceux qui sont pauvres et mauvais envient les riches, haïssent les bons, détestent leur gouvernement, en voudraient un autre et sont prêts à tout mettre sens dessus dessous. » Les idées de Burton sont sur ce point très proches de celles de Bacon dans l'essai sur les séditions et les troubles : «L'objet des séditions est double : la grande misère et le grand mécontentement », Essais, «Des séditions et des troubles », trad. Castelain, Aubier, 1979, p. 73.

23. «Beaucoup de gens refuseront de croire que de nos jours notre île de Grande-Bretagne n'est pas plus peuplée qu'elle l'était autrefois ; qu'ils lisent donc Bède le Vénérable [Histoire ecclésiastique], Leland [the laboriouse journey \& serche of John Leylande] et d'autres auteurs, ils se rendront compte qu'elle était des plus prospères à l'époque de l'heptarchie saxonne et à celle des conquérants normands, et qu'elle avait alors beaucoup plus d'habitants qu' aujourd'hui. Regardez donc le Domesday Book, et montrez-moi ces milliers de paroisses, aujourd'hui sans vie, ces cités en ruine, ces villages dépeuplés, etc. » («Au Lecteur», p. 14). Le dépeuplement est néanmoins davantage un mythe qu'une réalité, mythe qui disparaîtra lorsque la statistique fera son apparition.

24. More dénonce en effet dans le premier livre de L'Utopie les conséquences de ce mouvement dont sont responsables «les nobles, les riches, et même de très saints abbés »: « Ainsi un avare affamé enferme des milliers d'arpents dans un même enclos ; et d'honnêtes cultivateurs sont chassés de leur maison, les uns par la fraude, les autres par la violence, les plus heureux par une suite de vexations et de tracasseries qui les forcent à vendre leurs propriétés. Et ces familles plus nombreuses que riches [...] émigrent à travers les campagnes, maris et femmes, veuves et orphelins, pères et mères avec de petits enfants. Les malheureux fuient en pleurant le toit qui les a vus naître, le sol qui les a nourris, et ils ne trouvent pas où se réfugier. Alors ils vendent à vil prix ce qu'ils ont pu emporter de leurs effets, marchandise dont la valeur est déjà bien peu de chose. Cette faible ressource épuisée, que leur reste-t-il ? Le vol, et puis la pendaison dans les formes. Aiment-ils mieux traîner leur misère en mendiant? On ne tarde pas à les jeter en prisons comme vagabonds et gens sans aveu » (L'Utopie, dans Voyages aux pays de nulle part, présentation de Francis Lacassin, Laffont, «Bouquins », 2000, p. 128). 
d'exploiter les terrains communaux, contribuant du même coup à l'augmentation du prix du blé et du nombre de personnes privées de travail ${ }^{25}$. À l'inactivité des nobles risquent donc de s'ajouter l'inactivité des paysans et le nombre croissant de mendiants et de vagabonds qui envahissent les villes dans ce premier quart du XVII siècle.

Enfin Burton déplore l'état de restriction dans lequel se trouve le commerce ${ }^{26}$ ainsi que l'exploitation insuffisante des richesses dont dispose son pays. Le manque d'industrie oblige l'Angleterre à exporter ses matières premières et à racheter à prix d'or aux pays dotés d'une industrie efficace les produits ainsi transformés $^{27}$ :

[...] ils exploitent les chutes et en font des jouets et des babioles qu'ils nous vendent au prix qu'ils ont payé pour l'ensemble ${ }^{28}$.

Pour remédier à cet état de mauvaise santé, Burton propose deux remèdes : le travail et le développement de l'industrie et du commerce. L'analogie entre le corps naturel et le corps politique ne concerne pas seulement les symptômes et les causes de la mélancolie mais s'étend aussi à la question des remèdes. En

25. Cette analyse est remise en cause par les historiens qui insistent sur le fait que le mouvement des enclosure, sous le règne des premiers Stuarts, ne concernait que des superficies limitées, et qu'il était souvent réalisé avec l'accord des paysans. Voir F. J. RuGGIU, L'Angleterre des Tudors aux premiers Stuarts, Sedes, « Campus Histoire », 1998, p. 107.

26. F. J. RugGiu, op. cit., p. 115 : les premières années du règne de Jacques $\mathrm{I}^{\mathrm{er}}$ et la fondation de l'East India Company en 1600 marquent le début d'une période de fort développement commercial. Mais l'échec du Cockayne Project mis en place en 1614, qui visait à diminuer la dépendance économique de l'Angleterre par rapport aux ateliers flamands et hollandais, plonge le commerce extérieur anglais dans la dépression. À cela s'ajoutent le contexte politique et la guerre de Trente Ans.

27. «Nous avons [...] toutes sortes de matières, laine, lin, fer, étain, plomb, bois, \&tc., de nombreuses et excellentes idées sur la façon de les travailler, mais il nous manque l'industrie. Nous envoyons nos meilleures matières premières de l'autre côté des mers, où les gens les utilisent pour leurs propres besoins, les travaillent et les perfectionnent de diverses façons avant de nous les renvoyer à un prix plus élevé [...]» («Au Lecteur», p. 143-144). Sur cette question, voir F. Hermia DuRHAM, « The relation of the Crown to Trade under James I », Transactions of the Royal Historical Society, New Series, XIII (1899).

28. «Au Lecteur », p. 144. Ici encore, son propos est très proche de celui des mercantilistes et du Discours sur la prospérité publique de ce royaume d'Angleterre: «Je crois qu'il ne sort pas moins de cent mille livres par an de nos caisses, en échange d'objets sans aucune valeur intrinsèque, si ce n'est par le travail de leurs confectionneurs qui se procurent de l'ouvrage à nos dépens. Quelle balourdise est la nôtre de supporter cela, de nous laisser dépouiller continuellement de nos biens et de notre argent par des moyens semblables et spécialement de permettre à nos matières premières de quitter le pays, de devenir une occasion de travail pour les étrangers à qui nous les rachetons ensuite» (cité dans H. DENIS, op. cit., p. 121-122). Son diagnostic s'accorde aussi avec celui de Jacques $\mathrm{I}^{\mathrm{er}}$ dans le Basilikon Doron. Voir The Political Works of James I, introduced and edited by C. H. Mc Ilwain, Cambridge Mass., 1918, p. 26 : "They export necessary goods and import superfluous goods, if they import any at all », " they buy for us the worst wares, and sell them at the dearest prices ». 
effet, on peut appliquer selon Burton les mêmes remèdes à la mélancolie du corps naturel et à celle du corps politique : l'activité constitue pour Burton le meilleur remède contre la cause principale de la mélancolie, l'oisiveté. L'écriture de L'Anatomie de la Mélancolie est un remède contre la mélancolie de son auteur car elle constitue cet exercice indispensable qui permet à l'esprit d'échapper à l'oisiveté :

J'écris sur la mélancolie car je suis affairé d'éviter la mélancolie. La cause la plus puissante de la mélancolie est l'oisiveté, le meilleur remède est le travail... [P. 24.]

Le corps naturel, comme le corps politique, a besoin d'activité ${ }^{29}$ et de même qu'il «est impossible de préserver sa santé sans exercice physique », «il est difficile de s'enrichir sans travailler ni être industrieux $[\ldots]^{30} »$. Le remède imaginé par Burton (dont la principale vertu consiste à éliminer l'oisiveté du corps mélancolique) revient à faire du travail une véritable obligation : il faut « obliger les oisifs à travailler ${ }^{31} »$ : toute personne valide doit selon Burton, qui reprend sur ce point les thèses de More dans le livre II de L'Utopie ${ }^{32}$, se soumettre à cette obligation :

[...] je ne tolérerai pas la présence de mendiants, de rôdeurs, de vagabonds ou de toute personne oisive qui se révélerait incapable de justifier ses conditions de vie ou ses moyens de subsistance [...] ; s'ils en sont capables, ils seront obligés de travailler ${ }^{33}$.

29. Voir aussi BACON, Essais, p. 163 : «De la véritable grandeur des royaumes ». «Un corps, physique ou politique, ne saurait être en bonne santé que par l'exercice... ». Une « guerre honorable et juste » constitue selon Bacon une occasion d'exercice.

30. L'Anatomie de la Mélancolie, II.2.4.1. p. 855.

31. «Au Lecteur », p. 153.

32. Jean Starobinski montre bien dans son ouvrage sur l'Histoire du traitement de la mélancolie des origines à 1900, Documenta Geigy, Acta psychosomatica, Basle, Suisse, Geigy, 1960, p. 33-34) quelle a été la fonction thérapeutique du travail comme remède contre la mélancolie en particulier dans les écrits des pères de l’Église. «Priez et travaillez », tel était en effet le conseil adressé aux victimes de l'acédie. La fonction thérapeutique du travail consiste précisément à «boucher les brèches par où le démon pourrait pénétrer, par où la pensée oisive pourrait s'échapper ». Luther recommande lui aussi le travail comme moyen de résister aux assauts de la mélancolie, ce «bain du diable », qui rend l'homme vulnérable à la tentation. «Ainsi quand on sentira de ces pensées diaboliques, et qu'on se sentira tenté, voici mon conseil d'ami : pour chasser promptement ces idées, pensez à quelque chose de gai, ou buvez un bon coup, jouez ou amusez-vous, ou entreprenez quelque travail honorable auquel vous appliquerez, avec passion, toutes vos forces et vos capacités » (Martin Luther, Propos de table, trad. Louis Sauzin, Aubier, 1992, p. 233-234).

33. «Au Lecteur », p. 166-167. Rappelons que le statut des artisans de 1563 (en vigueur jusqu'en 1813) comporte l'obligation de travail et que la loi des pauvres de 1601 réprime le vagabondage. Voir H. DENIS, op. cit., p. 114. 
Pour parvenir à l'autosubsistance, Burton recommande l'exploitation des matières premières et interdit leur exportation ${ }^{34}$, mais il préconise surtout le développement du commerce et de l'industrie, en prenant comme référence le développement économique des Pays-Bas, champions du libre-échange au début du XVII ${ }^{e}$ siècle. Les Pays-Bas constituent en effet pour Burton le modèle à suivre en matière de développement économique ${ }^{35}$. Disposant de peu de matières premières, les Hollandais ont su néanmoins les exploiter « du fait de leur industrie, d'une politique intelligente et de leur commerce ». Burton oppose constamment dans la «Préface» la situation de l'Angleterre à celle des Pays-Bas : utilisant la fiction du voyageur qui irait observer «ces riches provinces unies de Hollande », il oppose «de belles cités et des villes fort peuplées, remplies d'artisans industrieux », des hommes capables de gagner des terres sur la mer et de les défendre au moyen de « digues artificielles d'une extraordinaire ingéniosité », des canaux navigables pour se rendre d'un endroit à un autre », à (du côté de l'Angleterre) des «milliers d'acres [...] inondés », des cités «malingres et laides, viles et pauvres», un «commerce ruiné», des «rivières hors d'usage », une absence de développement des modes de transport, des « villages dépeuplés ».

Tout en brossant ce tableau extrêmement sombre de la situation de l'Angleterre, qui correspond néanmoins à une réalité et ne peut pas être qualifié de pure invention de la part de l'anatomiste, Burton exprime en même temps cette conviction de la supériorité naturelle de son pays, que l'on retrouve dans les traités des marchands de l'époque, comme par exemple celui de Thomas Mun, qui écrit en 1622 :

Si nous considérons la beauté, la fertilité, la puissance maritime et terrestre de l'Angleterre [...] nous conviendrons que ce royaume est capable d'être maitre de l'univers, car quelle autre nation est si richement et naturellement dotée de choses nécessaires à la nourriture, à l'habillement, à la paix et la guerre, non seulement pour sa suffisance, mais aussi pour fournir ses voisins et en tirer chaque année abondance d'espèces et parachever sa félicité ${ }^{36}$.

34. Voir aussi p. 142: «Combien de cités magnifiques pourrais-je citer, qui ne vivent que de leur commerce et où des milliers d'habitants se nourrissent grâce au travail de leurs mains : Florence, en Italie, grâce à la fabrication du tissu d'or, la grande Milan, grâce à la soie et à d'autres ouvrages étranges, Arras, en Artois, grâce à ses belles tapisseries [...]. »

35. Voir p. 135, 137-138: « Mais j'admire par-dessus tout les Pays-Bas selon la description que nous en a donnée Lodovico Guicciardini. »

36. Cité dans C. H. Wilson, Cambridge Economic History of Europe, t. V, p. 518. Voir p. 136 : «C'est un pays riche et sanctifié, une des îles fortunées. À certains égards il est supérieur à d'autres pays, pour ce qui concerne le savoir de nos marins, le courage qu'ont demandé nos découvertes, l'art de la navigation, l'honnêteté de nos marchands... » 
Mais la véritable richesse d'un pays vient moins selon Burton de ses ressources naturelles que de son industrie et de sa capacité d'entreprendre :

Nous avons des moyens identiques, autant de possibilités physiques, des esprits ouverts, toutes sortes de matières premières, laine, lin, fer, étain, plomb, bois, \&tc., de nombreuses et excellentes idées sur la façon de les travailler, mais il nous manque l'industrie. [«Préface », p. 143.]

\section{USAGE DE LA MÉTAPHORE DU CORPS POLITIQUE ET DE LA THÉORIE DES HUMEURS CHEZ LES PREMIERS MERCANTILISTES}

Il y a donc de nombreux points de convergence entre le diagnostic établi par Burton dans la «Préface» de L'Anatomie de la Mélancolie en 1621 et les idées des premiers mercantilistes. Comme eux, Burton pense que la prospérité économique et le développement du commerce sont les conditions de la bonne santé de l'État, il est aussi persuadé du fait que l'Angleterre dispose de toutes les ressources nécessaires pour mettre fin à cet état de crise, et qu'il est nécessaire de faire appel à l'activité (business) pour remédier à l'oisiveté (idleness), la plus grande cause de la mélancolie individuelle mais aussi collective. Mais la richesse et la prospérité économiques ne sont des indices de la bonne santé du corps politique que si elles profitent à tous, car la pauvreté constitue un facteur de mécontentement et augmente les risques de sédition et de division du corps politique.

L'analogie entre la dimension individuelle de la mélancolie et sa dimension collective permet de construire un parallèle fort entre les causes, les symptômes et les remèdes à la crise mélancolique individuelle et ceux qui traduisent une crise politique : dans les deux cas, l'oisiveté a le statut de cause principale de la mélancolie; le déséquilibre (des humeurs ou la balance commerciale) peut être considéré comme un symptôme majeur de la crise et l'activité et le travail ont le statut de remèdes.

De leur côté, les mercantilistes font eux aussi usage de la métaphore du corps politique et de la théorie des humeurs pour défendre leurs idées : c'est-à-dire non seulement pour montrer que le développement du commerce est l'un des éléments constitutifs de la santé du corps politique et pour présenter la croissance économique comme un processus naturel dont la fonction consiste à assurer la bonne santé du corps politique, mais aussi pour insister sur la nécessité d'une régulation politique des échanges et d'une intervention de l'État dans le développement économique. Gérard de Malynes, par exemple, fait usage du discours 
médical et de l'analogie du corps politique dans son Treatise of the Canker of Englands Commonwealth ${ }^{37}$ (1601) pour penser le développement économique. Si le développement du commerce a dans son texte le statut de l'esprit vital (pneuma) chargé, d'après la médecine galénique, d'assurer la liaison de l'âme et du corps (the vital spirit of commerce), inversement tout ce qui fait obstacle à la prospérité économique peut être qualifié de désordre pathologique, et doit être expulsé ou purgé du corps politique. La principale maladie qui menace le corps politique est ainsi selon Malynes le déséquilibre de la balance commerciale, ce cancer, qu'il se donne pour tâche d'identifier et de soigner, suivant en cela la méthode des médecins qui consiste à identifier les causes de la maladie de manière à pouvoir proposer des remèdes (comme l'indique le titre complet de son traité : Traité du cancer qui ronge la République d'Angleterre, divisé en trois parties, où il est montré, en suivant la méthode des bons médecins, quelle est cette maladie, quelle est sa cause efficiente et quel remède on peut y apporter) : «Si nous sentons que le corps politique de l'État est déréglé [distempered] et plein de maladies, et si nous avons découvert les causes efficientes de ce dérèglement, pourquoi ne serions-nous pas capables de proposer des remèdes ? » demande Malynes dans le chapitre 5 d'un autre traité : The Center of the Circle of Commerce Com $^{38}$ (1623).

Une fois établi que la cause du dérèglement pathologique tient au déséquilibre de la balance commerciale, il reste à déterminer les moyens de rétablir l'équilibre. L'analogie du corps naturel et politique a comme principale fonction de justifier la nécessité d'une régulation politique du développement économique. Malynes fait une utilisation systématique de l'analogie qu'il présente dans le texte de 1623 sous la forme d'une allégorie : Allégorie du déclin du commerce en Angleterre, comparé à un corps naturel malade, avec les remèdes pour le soigner ${ }^{39}$.

Les hommes d'État et les hommes politiques ont comme première tâche, en tant que médecins du corps politique, de considérer l'état de santé dans lequel se trouve le corps politique du commerce (the politicke Body of Trafficque) et d'établir un diagnostic :

37. Gérard DE MALynes, A Treatise of the Canker of Englands Commonwealth, divided into three parts: Wherein the Author imitating the rule of good physitians, First declareth the disease, Secondarily, sheweth the efficient causes thereof, lastly, a remedy for the same, Imprinted at London by Richard Field for William Johnes printer, 1601.

38. Ce texte de Malynes est écrit en réponse et pour réfuter le traité de Misselden paru en 1623 : The Circle of Commerce. Son titre complet est le suivant: The Center of the Circle of Commerce or, A Refutation of a Treatise, Intituled The Circle of Commerce, or the Balance of Trade, lately published by E.M [Edward Misselden], by Gerard de Malynes, Merchant, London, 1623.

39. « An Allegory of the decayed Trade of England, compared to a diseased Body naturall : with the Remedies to cure the same », dans The Center of the Circle of Commerce..., chap. 5, p. 128. 
Les hommes d'État ou les politiciens (qui sont les médecins de la communauté) ayant sérieusement pris en considération l'état dans lequel se trouve le corps politique du commerce, constatent que ses parties externes sont pâles et blafardes, et diagnostiquent une consomption du foie ${ }^{40}$.

La cause de ce dérèglement est due à un déséquilibre de la balance commerciale (un excès d'importation de matières premières venant de l'étranger). Comment est-il alors possible de remédier à ce dérèglement? Tout d'abord, Malynes insiste sur le fait que la fonction de l'allégorie est de montrer qu'il faut avoir recours à des remèdes et que sans la médecine il est impossible de rétablir la bonne santé commerciale du corps politique. Il s'oppose clairement sur ce point à ceux qui, tout en reconnaissant la présence de la maladie, préconisent de laisser la nature agir, elle seule suffisant (sans aucune intervention de la médecine) à rétablir la bonne santé du corps politique. Le lecteur peut ici reconnaître la thèse de Misselden qui prône de son côté la liberté totale du commerce : il est inutile d'intervenir de l'extérieur pour rétablir l'équilibre de la balance commerciale dans la mesure où cet équilibre se rétablira naturellement en laissant les échanges se poursuivre librement ${ }^{41}$. Malynes se sert au contraire du discours médical pour montrer que l'intervention de l'homme d'État est nécessaire et que sans le recours à la médecine, le corps politique comme le corps naturel ne peuvent être guéris ${ }^{42}$.

Le recours au discours médical et à la métaphore du corps politique permet donc non seulement de décrire une situation de crise mais aussi de proposer des remèdes, tout au moins dans les ouvrages de Malynes. Le commerce a le statut de l'esprit vital qui circule à l'intérieur du corps politique et détermine sa bonne santé. Le développement économique est l'outil indispensable de la bonne santé du corps politique, à condition néanmoins que le magistrat, comme le médecin qui surveille l'équilibre des humeurs, soit capable de le contrôler.

Pourtant, si Burton partage avec les marchands de son époque cette conviction de la supériorité de son pays et de ses richesses potentielles qui lui

40. « An Allegory of the decayed Trade of England... », p. 128.

41. «Là-dessus, un certain empirique [empricke] (bien plus avancé que les doctes médecins) exposa sa thèse selon laquelle le corps en question (par un excès [surfeit] ou déséquilibre des matières premières étrangères) est tombé en consomption, mais que la nature est si forte, que (sans la médecine) elle recouvrera avec le temps son état premier de bonne santé » (ibid., p. 129).

42. «Si l'on prend en considération cette allégorie, l'on s'apercevra qu'elle contient une expression vivante des maladies du corps politique commercial [Politicke body of Trade] : et si un corps naturel avait toutes les maladies mentionnées il n'y aurait pas d'autre remède ou moyen de les soigner sinon en suivant la méthode qui a été ici exposée, comme en conviendra le médecin instruit par l'expérience : car les causes sont ici manifestes et évidentes, et il est impossible de les guérir si l'on ne fait pas appel à la médecine » (ibid., p. 130). 
permettraient de sortir de la crise, ce jugement entre en contradiction avec sa complexion mélancolique et son pessimisme concernant la capacité des hommes à se réformer.

\section{L'UTOPIE COMME REMÈDE À LA CRISE MÉLANCOLIQUE? MERCANTILISME ET UTOPIE}

D'un point de vue économique, l'on comprend donc quel peut être l'intérêt pour Burton de cette utilisation du discours médical. Le diagnostic proposé par les théoriciens mercantilistes quant aux causes de la crise économique et sociale que traverse son pays lui semble fondé, et le remède proposé par Gérard de Malynes (la régulation des échanges) s'accorde tout à fait avec la réforme économique qu'il appelle de ses vœux. En effet, il s'agit pour lui de parvenir à un compromis entre le modèle économique du libre-échange et la nécessité d'un développement économique juste : promouvoir le commerce tout en évitant les maux qu'engendre l'économie capitaliste naissante. Le développement économique est l'outil indispensable de la bonne santé du corps politique, à condition toutefois que l'État soit en mesure de le contrôler (Burton est partisan comme Malynes du système des étapes, staple system ${ }^{43}$, régulateur du commerce, de la fiscalité et de la protection du consommateur). Pour Burton, ce contrôle de l'économie répond à la fois à une exigence morale et à un souci politique : l'essor économique ne peut être considéré comme une condition de la bonne santé du corps politique s'il contribue à accentuer les inégalités entre riches et pauvres augmentant ainsi les risques de sédition et de soulèvement.

Mais jusqu'où la méthode proposée par les médecins pour guérir les maladies est-elle vraiment efficace lorsqu'on l'applique au corps politique ? Dans quelle mesure l'analogie du corps naturel et du politique permet-elle vraiment de proposer une réforme? « Ce ne sont pas des maisons mais des cités de correction qu'il nous faudrait ; la plus grande partie de notre commerce devrait être réformée ; il nous faudrait remédier aux manques », écrit Burton dans la «Préface » (p. 145). Mais ces souhaits, ajoute Burton, sont vains, et absurdes : attendre qu'ils soient exaucés est si ridicule qu'il faut les oublier : « tout doit rester tel quel $\gg$ (p. 153).

C'est alors sous la forme d'une utopie que Burton donne à voir à son lecteur une image de cet État non mélancolique qu'il appelle de ses vœux. Le changement est en effet impossible, dans la mesure où il suppose au préalable une

43. Cette exigence est de nature protectionniste. Voir le Staple Act de 1663. 
réforme morale qui concerne toutes les couches de la société (les hommes politiques, les médecins, hommes de loi, les philosophes, les marchands) :

Si c'était possible, je voudrais que les prêtres imitent le Christ, qu'ils soient des hommes de loi charitables et aimant leur prochain comme eux-mêmes [je voudrais] des médecins tempérés et modestes, des hommes politiques dédaignant le monde, des philosophes se connaissant eux-mêmes, des nobles vivant honnêtement, des commerçants abandonnant le mensonge et la fraude, des magistrats renonçant à la corruption, etc. Mais cela est impossible et il me faudra bien accepter le monde tel qu'il est ${ }^{44}$.

N'oublions pas que pour Burton, si le monde est mélancolique c'est parce que Dieu a envoyé ce fléau aux hommes pour les punir d'avoir péché. Dès lors, il n'est pas interdit de chercher des remèdes à cet état de fait, mais l'on ne pourra jamais le dépasser complètement. Cela n'empêche pas l'anatomiste de revendiquer la liberté d'imaginer ce que serait un État débarrassé de sa mélancolie, car dit-il : «Je dois veiller à ne pas peindre les choses trop en noir. » L'État imaginé par Burton répond précisément à la double exigence du développement des échanges et d'une régulation politique.

Partisan d'une organisation hiérarchique de la société ${ }^{45}$, Burton laisse la possibilité aux plébéiens de s'élever à la noblesse par le biais du travail et du mérite. Il réaffirme aussi la nécessité d'un gouvernement monarchique : "Ma forme de gouvernement sera monarchique ${ }^{46} »$, et se démarque des thèses de Thomas More en maintenant la nécessité de la propriété privée :

[...] tous les terrains seront enclos (sans toutefois aller jusqu'au dépeuplement $[\ldots])$; car ce qui est commun et à tout le monde n'est à personne ${ }^{47}$.

Il faut aussi laisser le commerce se développer, ce qui permettra d'occuper les oisifs en les obligeant à travailler, tout en soumettant le développement économique à un contrôle politique strict. Les échanges comme les prêts doivent être contrôlés ${ }^{48}$, les marchands doivent accepter le contrôle de leurs prix, les marchandises importées et destinées au plaisir et au luxe doivent être davantage taxées que celles qui répondent à des besoins primordiaux ${ }^{49}$. La vente de mar-

44. «Au Lecteur», p. 163 (souligné par nous).

45. «J'aurais divers ordres, divers degrés de noblesse, lesquels seront héréditaires [...] » (p. 160).

46. Ibid., p. 161.

47. Ibid., p. 158 .

48. «J'interdirai les courtiers, les prêteurs sur gage, les usuriers gourmands [...]. » « Je ne veux point de monopoles privés qui enrichissent un seul homme et réduisent la multitude à la mendicité » («Au Lecteur », p. 171).

49. «Mais tous les corps de métier devront accepter que le prix de vente de leurs produits soit 
chandises répondant à des besoins réels de la population doit par ailleurs avoir lieu dans les métropoles à des heures et des jours fixes, Burton interdisant les marchés et les foires ${ }^{50}$. Le dépeuplement, les monopoles, « l'altération de terres afforestées ou arables » ne peuvent être autorisés que sur l'ordre exprès de «certains inspecteurs qui auront été nommés dans ce but ${ }^{51}$ ».

De manière générale, Burton est favorable à l'établissement d'une société « hautement surveillée ${ }^{52}$ » où tous les aspects de l'existence des citoyens (depuis la naissance jusqu'à la mort, en passant par le mariage) sont contrôlés :

[...] j'instituerai des gouverneurs publics, des officiers habilités à chaque fonction, des trésoriers, des édiles, des questeurs, des syndics gérant le bien des orphelins et des veuves, et de tous les bâtiments publics $[\ldots]^{53}$.

C'est donc en instaurant un régime autoritaire et bureaucratique que l'on pourra supprimer la confusion et le désordre qui sont selon Burton les symptômes d'une mélancolie collective.

Le discours médical ne permet donc pas de penser jusqu'au bout une réforme économique. Il présente certes un intérêt considérable tant que l'on se situe au niveau de l'analyse des causes de la crise : il permet d'indiquer en un langage clair et accessible à tous, les symptômes du mal tout en dénonçant des abus inacceptables aux yeux de l'anatomiste. Mais l'analogie entre le corps naturel et le corps politique montre ses limites lorsqu'il s'agit de proposer des remèdes et une réforme : c'est pourquoi Burton l'abandonne pour construire une utopie. Comment expliquer cette cohabitation assez surprenante entre les thèses des mercantilistes et le discours utopique qui est, comme on peut le voir chez Thomas More (dont Burton reprend un certain nombre de critiques), plutôt critique à l'égard des conséquences du développement économique?

Tout se passe en fait comme si Burton avait conscience de la nécessité de réformer la société anglaise, mais ne souhaitait pas user de la métaphore orga-

évalué [...]. Les marchandises qui voyagent ou qui sont importées, lorsqu'elles sont nécessaires, pratiques \& importantes pour l'activité humaine, comme le blé, le bois, la houille, \&tc., ainsi que les marchandises absolument indispensables, ne devraient être que peu imposées, voire pas du tout, libres de taxes ; quant aux marchandises qui sont liées au plaisir, au luxe ou aux ornements, comme le vin, les épices, le tabac, la soie, le velours, le drap d'or, la dentelle, les joyaux, etc., celles-là devraient être imposées à un taux plus élevé » («Au Lecteur », p. 162).

50. Ibid., p. 155.

51. Ibid., p. 159.

52. Voir J. STAROBINSKI, op. cit., p. 66.

53. «Au Lecteur», p. 158. J. Starobinski fait remarquer que ces « superviseurs » sont les descendants directs des «Syphograntes » imaginés par Th. More au second livre de L'Utopie, personnages dont la principale fonction consiste à surveiller que l'obligation de travailler est bien respectée et que personne ne s'adonne à l'oisiveté. 
niciste de manière à exprimer la nécessité d'un changement effectif et radical. Burton reste fondamentalement un partisan de l'ordre établi. Sa position est conforme à celle des pasteurs anglicans orthodoxes qui considèrent que le changement est plus dangereux pour un organisme que le déclin naturel. Sa position semble être de dire que la seule manière de s'acheminer vers la guérison consiste à prendre acte de cette dégénérescence irréversible du corps politique : la crise mélancolique doit logiquement conduire au déclin puis à la mort de ce corps. C'est là une thèse que l'on trouve par exemple sous la plume du pasteur anglican Calybute Downing qui présente, dans un traité de 1634, le repos et la quiétude comme les seuls remèdes possibles à la maladie du corps politique, refusant d'envisager une quelconque rupture ou changement :

Or tout changement qui dérange est dangereux, particulièrement lorsque le corps de la république est rempli de maladies ${ }^{54}$.

Ce constat, Burton semble le faire sien lorsqu'il déclare que tous les souhaits qu'il vient de formuler et qui permettraient de guérir l'Angleterre de sa mélancolie sont vains et que « tout doit rester tel quel» (p. 153). Certes, «le monde se transforme tous les jours [...]», mais la folie, elle, est incurable : «Les langues, les habitudes, les lois, les coutumes, les manières sont soumises à des changements, mais pas les vices, ni les maladies, ni les symptômes de la déraison ou de la folie - ceux-là restent inchangés ${ }^{55}$. »

Dès lors, seule la fiction peut avoir des vertus curatives : c'est pourquoi Burton imagine un État dont la forme traduit l'exigence puritaine d'une activité continue, organisée, méthodique, et qui permettrait de bannir l'oisiveté en remédiant à la confusion qui s'est introduite dans le corps politique avec le mouvement des enclosures et la dislocation de l'ancienne société. Burton appelle de ses vœux une société dans laquelle la corruption des princes et des hommes d'Église aurait disparu, où chaque citoyen travaillerait dans l'intérêt de la communauté et non pas pour son seul profit, où l'impunité n'existerait pas, où les échanges et tous les actes des individus seraient soumis à un contrôle strict destiné à les empêcher de suivre leurs passions pour se conformer à l'usage de la raison. Mais ce changement radical ne peut prendre que la forme d'une utopie, dont la seule fonction est d'aider le mélancolique qu'est Burton à surmonter sa maladie. C'est peut-être encore une fois la mélancolie dont il souffre lui-même qui le persuade que tout changement est impossible, et qui le conduit à donner à la guérison la forme d'une fiction. L'utopie imaginée par Burton n'a pour unique

54. Calybute Downing, A Discourse of the State Ecclesiastical, Oxford, 1634, p. 14-15. 55. « Au Lecteur », p. 77. 
but que sa propre satisfaction et son propre plaisir, exactement comme les délires imaginatifs des mélancoliques répondent à une situation de crise personnelle et les aident à la dépasser ${ }^{56}$.

Claire CRIGNON-De OLIVEIRA

École normale supérieure

Lettres et sciences humaines, Lyon

56. Voir sur ce point les analyses de W. SCHLEINER, Melancholy, Genius and Utopia in the Renaissance, O. Harrassowitz, 1991, p. 203. 